\title{
THE PULLER-FOLLOWER CONTROL CONCEPT IN THE MULTI-JOINTED ROBOT BODY WITH ANTAGONISTICALLY COUPLED COMPLIANT DRIVES
}

\author{
Veljko Potkonjak*, Kosta M. Jovanovic*, Predrag Milosavljevic*, Nenad Bascarevic*, Owen Holland ${ }^{\text {II }}$ \\ *Faculty of Electrical Engineering, University of Belgrade \\ Bulevar kralja Aleksandra 73, 11000 Belgrade, Serbia \\ potkonjak@yahoo.com, kostaj@etf.rs,.mp103041m@student.etf.rs, bn103038m@student.etf.rs

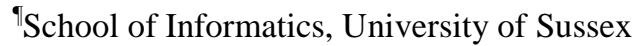 \\ Brighton BN1 9 QJ, Falmer, United Kingdom \\ owen.holland@gmail.com
}

\begin{abstract}
Copying human physiology leads us to the first truly anthropomimetic robot - ECCEROBOT, driven by the antagonistically coupled compliant drives. Control design of such a mechanism appears as a really demanding and challenging mission. Puller-follower concept, developed for the robotic joint with antagonistically coupled drives, is expanded to the multi-joint control level. Problems in control of the multi-jointed anthropomimetic robot are highlighted in this paper, and solutions through the robust control and model based compensations are proposed.
\end{abstract}

\section{KEY WORDS}

Bio-inspired robotics, antagonistic drives, non-linear control, passive compliance.

\section{Introduction}

The idea of this paper is mainly based on the current work within the FP7 project "Embodied Cognition in a Compliantly Engineered Robot", at the Faculty of Electrical Engineering, University of Belgrade as one of the partners. ECCEROBOT [1] is the one advanced humanoid robot with the great accent to inner similarity to human being. Both skeleton and musculature are copied using new materials and innovative drive structures. Knowing robot structure and its final aim making artificial man [2], control problems appears as the inevitable and the most important task. ECCEROBOT's artificial muscles are antagonistically coupled, nonlinear and compliant drives, consisting of non-elastic thread, spring and DC motor equipped by gearbox and real. This work is based on the previous experience in the field of control of such a drives, but this time control task is extended to the multi-joint level. Considering multijointed system, new effects appeared and they are pointed out in this paper. Already elaborated puller-follower concept for the single joint [3] with certain changes is applied in order to deal with these effects, but also some new solutions are suggested.
In Section 2, the latest ECCEROBOT prototype is presented, and brief insight in his structure is given, emphasizing advantages of this kind of robots. Demanding control mission of the robot joint with antagonistically coupled compliant drives using pullerfollower concept is explained in Section 3. Section 4 points out effects appearing in the multi-jointed robotic system, that we should be aware of. Dealing with these effects, Section 5 describes our solutions and suggestions in control through the gravity compensation, robust control and modification of the puller-follower concept. Achieved results are presented in the Section 6, demonstrating robot karate move - "inside block chudan uchi uke".

\section{Multi-jointed robot model with antagonistic drives}

The most advanced humanoid robots are made according human body frame and appearance, but their mechanisms are very different from those in humans $[4,5]$. Although quite complex structure, these robots still use well known and explored stiff and heavy drives, also widespread in industrial robots application. Contrary, human body is highly compliant system with muscles which have power to weight actuation ratio far above this currently actuators.

Not so many projects in the field of antropomimetic robots, embodied cognition and related topics have been presented so far. One of these is the $\mathrm{iCub}$ representing three and a half year old child about $104 \mathrm{~cm}$ tall and about $22 \mathrm{~kg}$ weight. iCub robot has 53 degrees of freedom (DOF) partly with conventionally actuated joints [6]. However, some of joints (hands, ankles, shoulders, waist) are tendon-driven joints consists of brushless motor in series with cable which are inelastic. The most similar project to our ECCEROBOT project is Tokio JSK laboratory's robot Kojiro. Kojiro project regards benefits of this anthropomimetic approach and modern technology in practical robotics applications [7]. 
The ECCEROBOT constructor's idea was to create the robot, the most similar ever to human upper body, fixed on the mobile base (Fig. 1), by not just replicating the appearance of the human, but also duplicating the inner structures and mechanisms of humans: bones, joins, muscles and tendons. Robot bones are made of polymorph, new type of thermoplastic which has bone like appearance when it is cold. Each ECCEROBOT joint is driven by the two antagonistically coupled artificial muscles similar to the most of the human muscles responsible for movements, instead of high precision actuators and stiff components as in typical humanoid robots. These muscles are introduced as the combination of non-elastic threads, elastic tendons and DC motors equipped with gear-boxes. Thus, desirable mechanical compliance is achieved by using elastic spring for connecting non-elastic thread to the robot links, copying human muscle and tendon. Following the anthropomimetic principle [8], ECCEROBOT is aimed to be first one with the great dose of self consciousness.

Starting point for the work in the field of control was dynamic model development for the joint types, as well as the development of the complete dynamics robot model

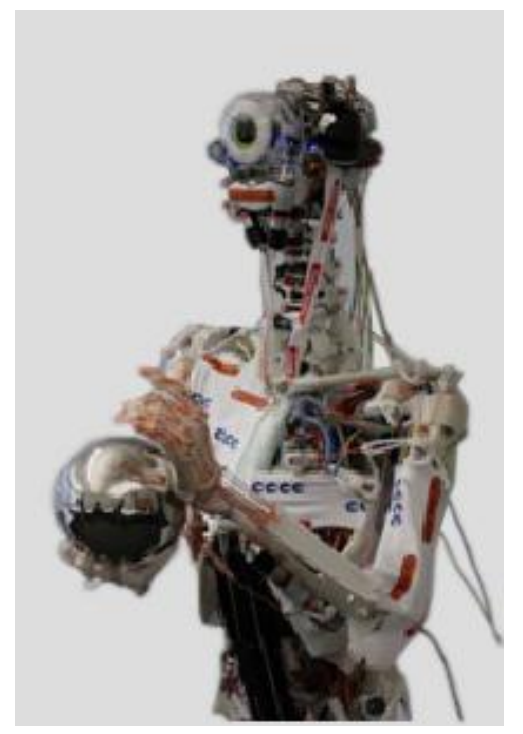

Fig. 1. The latest prototype of the ECCEROBOT

including antagonistically coupled compliant drives. This is done in [9].

Robot dynamics is described by (1), relating appropriate driving torques to the robot movement (without importance of how these torques are generated).

$$
\mathbf{H}_{n x n}(\mathbf{q}) \ddot{\mathbf{q}}_{n x 1}+\mathbf{h}_{n x 1}(\mathbf{q}, \dot{\mathbf{q}})=\boldsymbol{\tau}_{n x 1}
$$

Where $\mathbf{H}_{n x n}$ is the inertial matrix, and $\mathbf{h}_{n x 1}$ takes care of gravity, centrifugal, and Coriolis' effects, and $\boldsymbol{\tau}_{n x 1}$ is driving torque vector.

\section{The puller-follower concept - control of the antagonistically coupled drives}

Even though the advantages of the anthropomimetic design are obvious, there are still challenges in controlling the robot. Simple movements like lifting an arm, requires the actuation of various muscles and all together with its compliant nature indicate control task as really challenging and demanding mission.
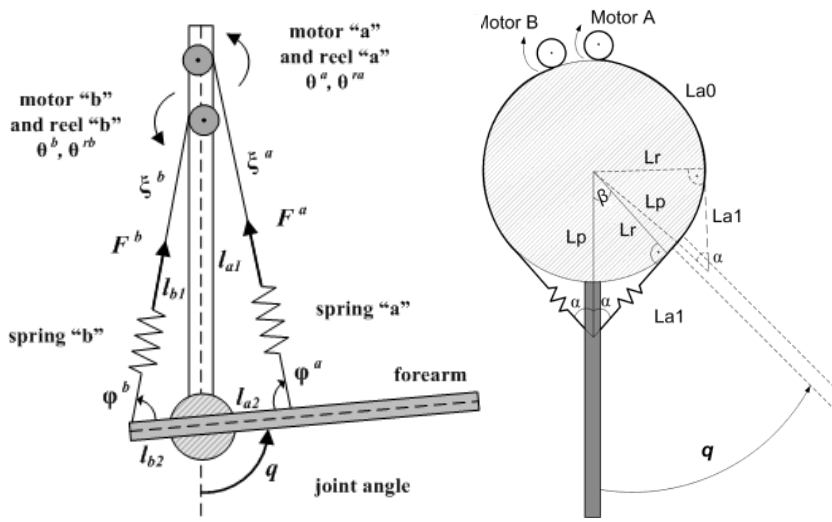

Fig. 2. Elbow rotation with AA drives - triangular model (left)

Shoulder rotation with AA drives - circular model (right)

Considering ECCEROBOT structure we have adopted two different models of the robot joints (Fig. 2) that suit the real joints. The first one is triangular joint which is intuitive representation of the elbow joint in humans. What is more this agonist-antagonist (AA) pair biceps-triceps, is the most illustrative example of the antagonistically coupled muscles in human beings. However, this model hardly matches other joints of the ECCEROBOT, so we designed the new model - circular joint model. The circular joint model is suitable as approximation of the most others joints with antagonistic drives.

There are a few authors who have already considered control of the robot joint with antagonistic drives, also a great number of them considered benefits of the compliant joints. However, they usually explored different joint structures, and their improvements for the possible later use. Our problem was a little different. We have already had exact structure and we should have face with control issue. The main problem of controlling an electrical AA drive lies in the antagonistic drive's redundancy. One motor, the agonist, initiates the action, while the other, the antagonist, opposes it. In order to achieve a desired joint motion, the motors should be driven simultaneously, in a coordinated way. We also have to consider the tendons coupling the motors to the joint. This tendon coupling enforces unidirectional power transmission and, without the correct control, the tendons can slacken, leading to an undesirable backlash in the torque transmission.

We have faced these problems gradually. In the very beginning we observed simplified systems moving to the 
real target configuration - revolute, non-liner and compliant robotic joint (Fig. 2). We started from the linear, non-compliant system (Fig. 3) dealing with just tendon coupling and drive redundancy (antagonism).

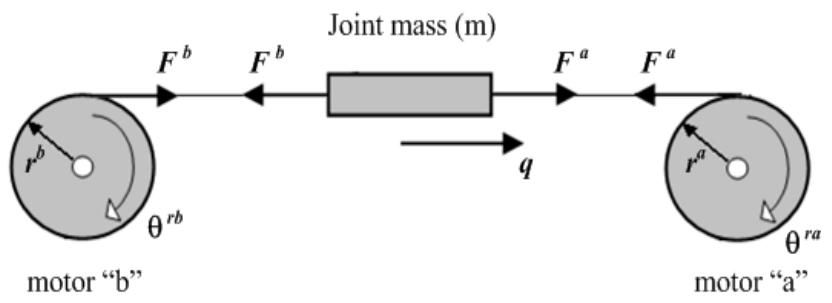

Fig. 3. Linear, non-compliant robotic joint

Then we added compliance by adding elastic springs to the non-elastic ropes making linear, compliant robotics joint (Fig. 4). Doing this, control problem of the passive compliance (elasticity) appeared. What is more, by adding compliance number of degrees of freedom in joint is tripled.

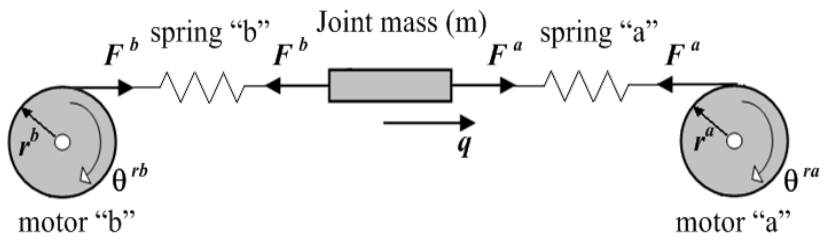

Fig. 4. Linear, compliant robotic joint

Gradually analysing emphasised effects, control problem was considered in [3].

Eventually, in order to deal with this complex control problem, we developed a completely new biologicallyinspired and energy efficient approach - the pullerfollower concept. Idea of this concept has its origin in the biological systems of antagonistically coupled muscles in human being. The basic control requirement is the control of the joint position. One motor, the puller, takes the main responsibility for this task. In order to prevent slackening of the antagonist tendon, a second control task maintaining an appropriate tension in the tendon - is added. This additional task is mainly assigned to the follower motor. By "appropriate tension" we mean a tension above some minimal value that guarantees that a tendon will remain stretched during joint motion. This prescribed minimum will be called the reference tension force. We rely on a multivariable feedback approach [10] - input-output feedback linearization for multiple-inputmultiple-output (MIMO) systems [11]. This method counteracts the nonlinearity [12] and interactions in the model and transforms the original two-input-two-output (TITO) system into two decoupled single-input-singleoutput (SISO) systems. By adopting this approach, we have to solve similar control problems for both joint models. Higher control logic is then applied in the form of four SISO controllers (one position and one force controller for each of the motors) and a switching logic block. Therefore, the two SISO controllers are active all the time. Accordingly, in each joint we will have two references: the reference position (i.e. the reference motion trajectory) and the reference tension force.

The joint motors exchange roles when the motion requires it, usually when acceleration turns to deceleration. This exchange of roles is called switching. The reference tension force is not kept constant during the motion, and it is increased shortly before switching, and it is reduced after it. The reason for introducing this adaptive reference force comes from the observation that switching often causes a negative overshoot of the tendon force in the transient period after switching.

The great importance is given to the relation between pulling and following force, observing energy efficiency on the one side and the undesirable effect leading to slackening of the rope on the other. These undesirable effects are oscillations in transient phase and negative overshot during switching which jeopardise system behaviour. Although this overshoot is relatively small in magnitude, the small reference value can lead to the pulling force (now becoming the following force) falling below zero, i.e. the tendon becomes slack. We have proved [13] that slackening can be prevented by using a higher value for the reference tension force. To avoid an increase in energy consumption, we raise the force reference shortly before switching and reduce it immediately after the transient oscillations end. This makes our approach energy efficient.

Let us be more precise and about the influence of the reference tension to the system dynamics after switching. Our simulations revealed that the switching shock depends strongly on the level of the reference tension force, as shown by the dotted line in Fig. 5. It can be seen that the minimum shock appears when the reference tension force is about $25 \%$ of the actual pulling force. We also examined the minimum values of the tension forces during switching (shown by the dashed line in Fig. 5).

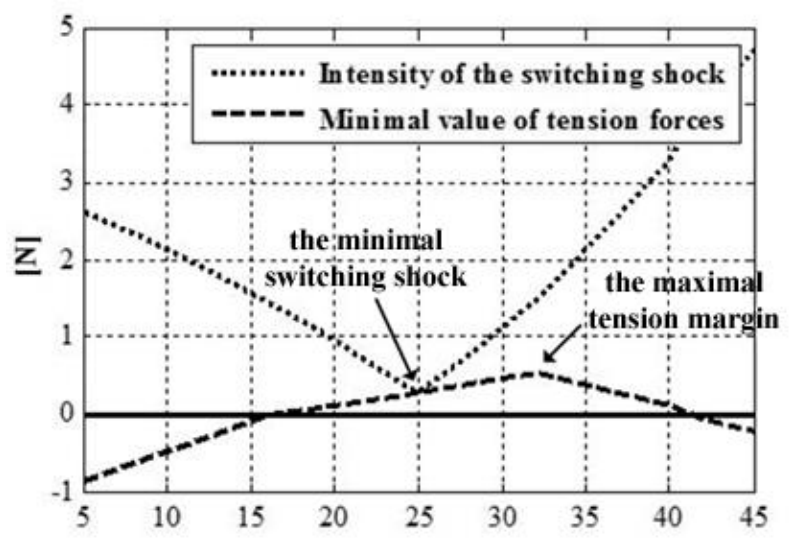

The switching shock as a proportion of the pulling force [\%]

Fig. 5. The trade off between the minimal switching shock and the maximal stretching margin 
It can be seen that if we raise the reference tension force from $19 \%$ to $40 \%$ of the actual pulling force, then both tension forces will be positive (i.e. both tendons will be taut) all the time. Here we introduce the idea of the maximal tension margin, which is the value of the reference tension force (as a percentage of the actual pulling force) at which the minimum value of the tension forces during switching is highest. This occurs when the reference tension force is close to $32 \%$ of the pulling force. Therefore, we can choose the reference tension in order to gain either of two advantages: the minimal switching shock (with the reference tension set to $25 \%$ of the actual pulling force) or the maximal tension margin (with the reference tension set to $32 \%$ of the actual pulling force).

Briefly, switching occurs when puller-follower concept is trended to be threatened. In these moments the puller motor (and the corresponding cord) comes in to the situation to "push" or to get pulling tendon slacken. To avoid the unacceptable state, the switching logic triggers and the other motor starts to pull. We have developed a new idea of fuzzy adaptive reference force that activates under the next conditions:

- the slope function of puller force is higher than $70^{\circ}$

- the value from which puller force function starts rapidly to decline is higher than $200 \%$ of tension reference force value

- the reference force will be increased if puller force reaches the value of tension reference force

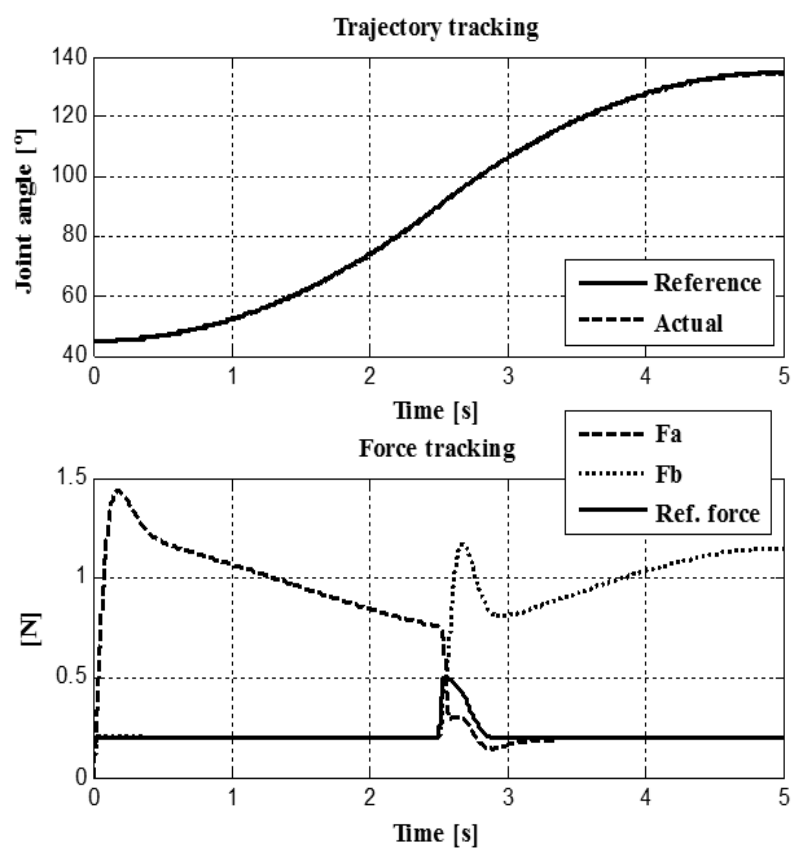

Fig. 6. Trajectory tracking and force tracking in a triangularmodel joint
Fig. 6 illustrates puller-follower concept applied to the triangular joint model. Although the displayed motion in horizontal plane is slow in order to demonstrate logic, trajectory tracking seems very good for also demanding and faster movements. During the movement in horizontal plane according to the triangular velocity profile, exchanging the roles between two motors are needed at the half interval. Thus pulling force $(\mathrm{Fa})$ caused by the motor A for the position control, becomes the following responsible for force tracking and vice-versa for motor B. Fig. 6 also points out switching zone where the adaptive tension force prevent oscillations and switching shock in transient phase and therefore prevent slackening of the ropes.

\section{Overview of the dynamic effects in the multi-jointed anthropomimethic robot}

Starting from the antagonistically coupled linear noncompliant joint, and gradually adding compliance in the first and then nonlinearity in the second step, control algorithm for the triangular and circular joint is developed. Based on multivariable nonlinear control theory desirable behaviour of the isolated robot joint is achieved. Our approach needs motor and drive parameters (what can be found in the catalogues or they can be easily identified through the simple experiments), but also link parameter such is its mass and shape (actually moment of inertia).

The idea of our research is to determine to what extend our control concept can be applied to the ECCEROBOT. Thus, we move to the multi-jointed robotic system, and tested our algorithm on the complete system. From the very beginning many problems appeared. Next paragraph lists this effects we realized.

Effective proper inertia - For a particular joint, the effective moment of inertia depends on all other links in the kinematic chain, not only the following segment. For example, effective moment of inertia in shoulder joint is several times different if we move hand with flexed or extended elbow position. Particularly, full flexing of the elbow causes four time lower moment of inertia.

Dynamic coupling between the joints - This includes some inertial effects, which can strongly influence the control of the single joint. For example, just keeping the single joint position can be demanding task, if the other joints in the same chain make intensive movements.

Gravity force - Depending on the robot position the same joint can work in the horizontal or vertical plane. Working in the horizontal plane the gravity force does not influence the joint behaviour, and require very low tendon forces. On the other hand, the same move in the vertical plain requires several times stronger tendon forces due to gravity. What is more, due to gravity our antagonistic motors working as the puller or the follower could have the different roles during the same move in the horizontal and in the vertical plane. So, lifting an arm in vertical plane can be achieved without switching the roles 
(depending of the speed of acting), while that move can require several switching in the horizontal plain.

\section{Control concept of the multi-jointed robot with antagonistically coupled drives}

We have examined the extent to which the single-joint control strategy can be applied to this multi-joint system through the ECCEROBOT hardware architecture [TUM], and brief conclusion is given in this Section.

The solution we propose for this problem is a robust control system design in a combination with our nonlinear control strategy. We will design the robust controller in order to deal with the uncertain parameters and the unmodeled dynamics that are presented in the single-joint system, when it is implemented in the multi-joint system. Because of applied input-output feedback linearization, uncertainty in the system cannot be described as a function of system parameters and the only robust control design method that can be used here is $H_{\infty}$ loop-shaping. Changeable gravity force influence can be determined from the model of the spatial system, and the nonlinear gravity compensation is introduced on this side, as it will be presented later in the text.

\subsection{Gravity compensation}

The aim of this subsection is to make control system aware of gravity influence and to define proper compensation. In order to take care of the fact that the gravity load of a joint depends on the positions of the other joints, we start from the multi-joint system and extract the gravity component from the model (1). The vector $\mathbf{h}(\mathbf{q}, \dot{\mathbf{q}})$ includes the static gravity load $\mathbf{h}_{g}$ and the dynamic velocity-dependent effects $\mathbf{h}_{d}$ (centrifugal, Coriolis', and viscous friction):

$$
\mathbf{h}(\mathbf{q}, \dot{\mathbf{q}})=\mathbf{h}_{g}(\mathbf{q})+\mathbf{h}_{d}(\mathbf{q}, \dot{\mathbf{q}})
$$

To control a particular joint $-j$, we use a single joint notation where the gravity moment is expressed as:

$$
G_{j}=h_{g_{j}}(\mathbf{q})
$$

One of the possible solutions for gravity compensation is to determine the modified feedback linearization for the system including the gravity component. This changes the corresponding values in the decoupling matrix, thus making the control system aware of the influence of gravity. Similar research is carried out in [15].

\subsection{Robust control}

In this subsection the influence of the uncertain moment of inertia $I$ of robotic chain on the system behaviour is examined. A linear robust control, which guaranties the robust stability and the robust performance of the closed loop system, will be designed. $H_{\infty}$ loopshaping design procedure consists of two steps. First the linear controller $K_{s}$ is designed, which provides desired performances, and it is used as a pre-compensator $W_{1}$. For the post-compensator we choose $W_{2}=1$. Controller structure is shown in Fig. 7. After loop-shaping, $K_{\infty}$ controller is computed which provides robust stability. Final controller is therefore given with $K=W_{1} K_{\infty} W_{2}$.

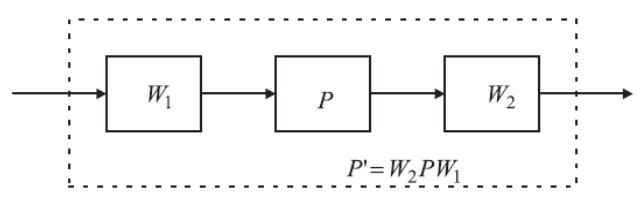

Fig. 7. Loop shaping: $\mathrm{W}_{1}$-pre-compensator, $\mathrm{W}_{2}$-postcompensator, P-plant

Therefore we have two systems that we should take care of - position and force. First, the position controller $K_{p}$ is determined and then the force controller $K_{f}$. Controller $K_{p}$ corresponds to the SISO system $G_{p}{ }^{I}(s)=$ $1 / s^{4}$ and controller $K_{f}$ corresponds to the SISO system $G_{f}{ }^{I}(s)=1 / s^{2}$. The robust stability and the robust performance in single-joint system was achieved for any value of the effective joint inertia $I$ in the range $\left[0.5 I_{\text {nom }}\right.$, $\left.3 I_{n o m}\right]$ and for any influence of gravity $i_{g}=[0 \%, 100 \%]$. But, when we checked these controllers in the multi-joint system the control performance was compromised. Additionally, we have to take into consideration that the motors' input voltage is limited to $12 \mathrm{~V}$. Therefore, control signals that come from regulators must not be higher than mentioned voltage value for some longer interval of time. In opposite, the saturation effect in actuation will appear. In order to deal with this problem, we suggested several position and force controllers, having different bandwidth, which were robustly tuned. Namely, we try to satisfy next requirements (due to loop shaping control methods):

- big gain on low frequencies;

- fast decreasing gain after bandwidth frequency;

- $20 \mathrm{~dB} / \mathrm{dec}$ slope at the bandwidth frequency;

- phase margin more than $40^{\circ}$. 

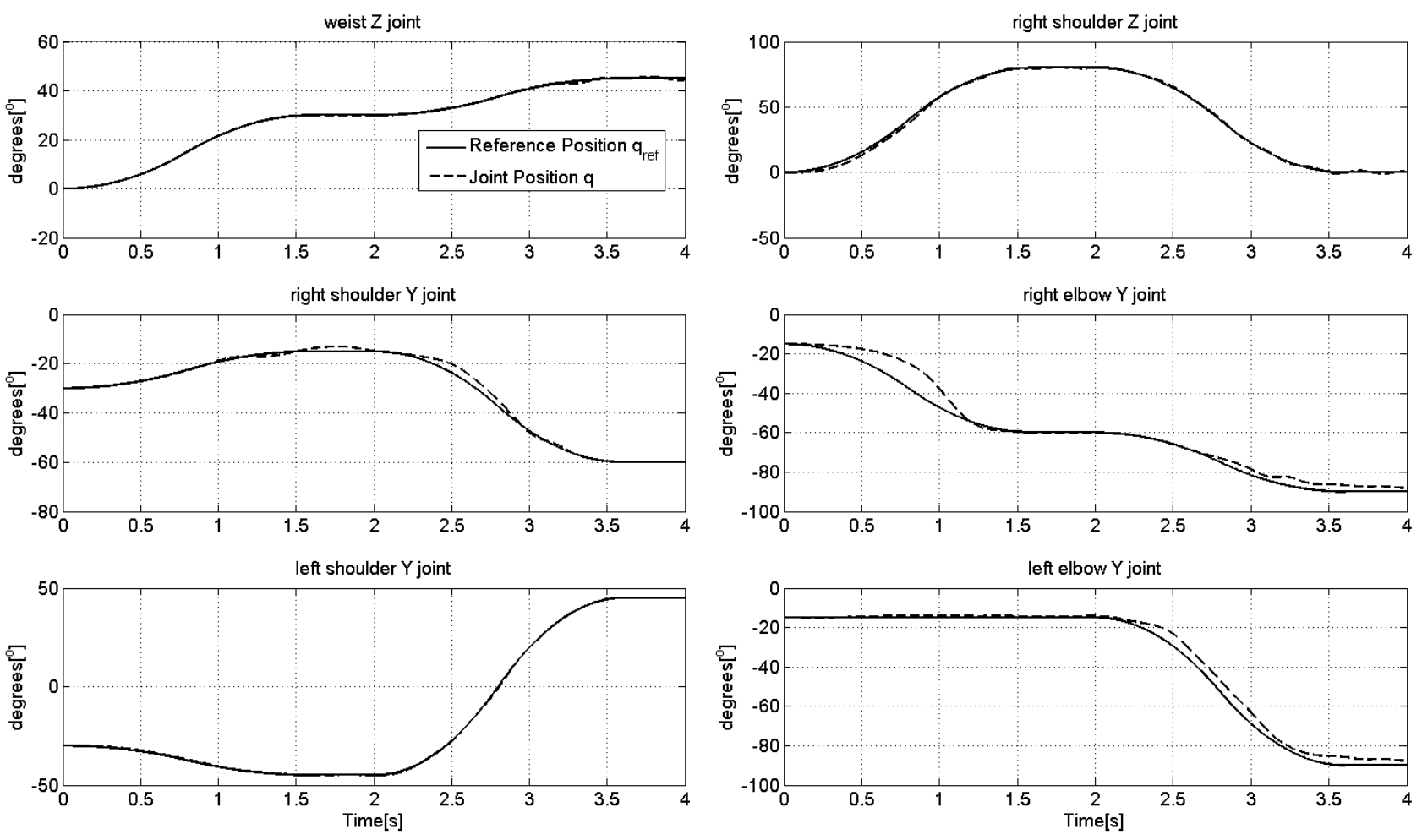

Fig. 8. Joint positions during the Uchi-Uke karate move

\section{Conclusion}

With its compliant human-like shape, such a mechanism is able to interact with humans and with its environment in an inherently safe way. So far, results of our algorithm based on the multivariable input-output feedback linearization, $H_{\infty}$ loop-shaping robust control and gravity compensation, are presented through the inside block Chudan Uchi-Uke karate move. This move is chosen as attractive one and prime example of the movement with strong dynamical coupling between the joints. Also big changes in the effective proper inertia in the joints are presented during the move. Joint positions realizing the main action during this karate move are shown in Fig. 8. We can notice small position error in the Fig. 8, but conclusion is that acceptable behaviour is achieved. The most illustrative examples of the control including switching the motor roles and adaptive tension forces are displayed in the Fig. 9.

Final aim of the project is to design control based on the symbiosis of our approach using engineering control techniques and the other approaches using artificial intelligence algorithms. So far, there is no such a perfect control of a so advanced humanoid mechanism like the human brain. However, the advantages of the design prevail and we are very confident that once the control problem is solved, this structure will propel the concepts of embodiment and will pave the way for a new era in robotics.
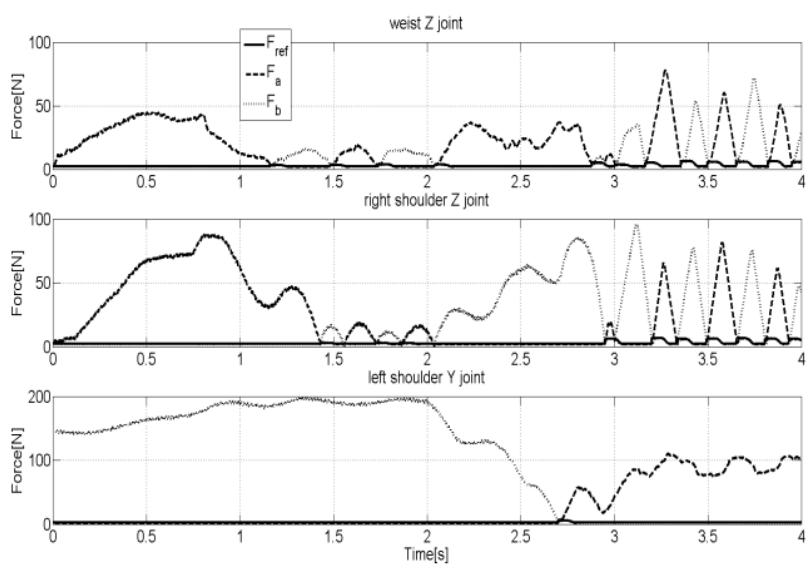

Fig. 9. Tendon forces during the Uchi-Uke karate move

\section{Acknowledgements}

The research leading to these results has received funding from the European Community's Seventh Framework Programme FP7/2007-2013 - Challenge 2- Cognitive Systems, Interaction, Robotics - under grant agreement no. 231864 - ECCEROBOT; and partly by the Serbian Ministry of Science and Technological Development under contract 35003. 


\section{References}

[1] H. G. Marques, M. Jäntsch, S. Wittmeier, C. Alessandro, M. Lungarella, R. Knight, O. Holland, ECCE1: the first of a series of anthropomimetic musculoskelal upper torsos, Proc. of Humanoids (Humanoids 2010) Nashville, TN, USA, 2010, in press

[2] T. Fukuda, R. Michelini, V. Potkonjak, S. Tzafestas, K. Valavanis, M. Vukobratovic, How Far Away is "Artificial Man", IEEE Robotics and Automation Magazine, 2001, 66-73.

[3] V. Potkonjak, B. Svetozarevic, K. Jovanovic, O. Holland, Control of Compliant Anthropomimetic Robot Joint, Proc. International Conference on Numerical Analysis and Applied Mathematics (ICNAAM 2010), Rhodes, Greece, 2010, 1271-1274.

[4] Y. Sakagami, R. Watanabe, C. Aoyama, S. Matsunaga, N. Higaki, K. Fujimura, The intelligent ASIMO: system overview and integration, Proc. International Conference on Intelligent Robots and Systems (IROS 2002), Lausanne, Switzerland, 2002, 2478 $-2483$.

[5] T. Ishida, Development of a small biped entertainment robot QRIO, Proc. of International symphosium on Micro-Nanomechatronics and Human Science (MHS 2004), and the Fourth Symposium on Micro-Nanomechatronics for Information-Based Society, Nagoya, Japan, 2004, 23-28.

[6] G. Metta, G. Sandini, D. Vernon, L. Natale, and F. Nori. The iCub humanoid robot: an open platform for research in embodied cognition, in PerMIS: Performance Metrics for Intelligent Systems Workshop, Washington DC, USA, 2008.

[7] I. Mizuuchi, Y. Nakanishi, Y. Sodeyama, Y. Namiki, T. Nishino, N. Muramatsu, J. Urata, K. Hongo, T. Yoshikai, and M. Inaba An advanced musculoskeletal humanoid Kojiro, Proc. of International Conference on Humanoid Robots (Humanoids07), 2007, 294-299.
[8] O. Holland, R. Knight, The Anthropomimetic Principle, Proc. of the Symposium on Biologically Inspired Robotics edited by J. Burn and M. Wilson (AISB06), Bristol, UK, 2006

[9] V. Potkonjak, B. Svetozarevic, K. Jovanovic, O. Holland, Biologically-inspired control of a compliant anthropomimetic robot,Proc. of the 15th IASTED International Conference on Robotics and Applications (RA 2010), Cambridge, Massachusetts, USA, 2010, 182189.

[10]S. Skogestad, I. Postlethwaite, Multivariable Feedback Control (John Wiley \& Sons Ltd., England, 2005).

[11] G. Palli, C. Melchiorri, A. De Luca, On the feedback linearization of robots with variable joint stiffness, Proc. of the IEEE International Conference on Robotics and Automation (ICRA 2008), Pasadena, California, USA, 2008, 1753-1759.

[12]H. K. Khalil, Nonlinear Systems, Third Edition (Upper Saddle River, New Jersey, Prentice Hall, 2002).

[13] V. Potkonjak, B. Svetozarevic, K. Jovanovic, O. Holland, Anthropomimetic Robot with Passive Compliance - Contact Dynamics and Control, Proc. of the IEEE 19th Mediterranean Conference on Control and Automation (MED '11), Corfu, Greece, 2011, 1059 1064.

[14] M. Jäntsch, S. Wittmeier, A. Knoll, Distributed control for an anthropomimetic robot, Proc. of the IEEE/RSJ International Conference on Intelligent Robots and Systems (IROS 2010), Taipei, Taiwan, 2010, 5466-71. [15] A. De Luca, B. Siciliano, L. Zollo, PD control with on-line gravity compensation for robots with elastic joints: Theory and experiments, Automatica, 41, 2005, 1809-1819. 